\title{
Developing a Real-Time Web Questionnaire System for Interactive Presentations
}

\author{
Yusuke Niwa, Shun Shiramatsu, Tadachika Ozono, Toramatsu Shintani \\ Department of Computer Science, \\ Graduate School of Engineering, Nagoya Institute of Technology \\ Gokiso-cho, Showa-ku, Nagoya, Aichi, 466-8555 Japan
}

\begin{abstract}
Conducting presentations with bidirectional communication requires extended presentation systems with sophisticated expressions and real-time feedback gathering. We aim to develop an interactive presentation system to enhance presentations with bidirectional communication during presentations. We employed a hybrid approach of a traditional presentation supporting system (PowerPoint) and a Web application to realize the interactive presentation system. The audience can provide direct feedback on delivered slides using the Web applications; then, the feedback is synchronized to the presenter's slides. Specifically, the presenter collects opinions using our real-time questionnaire function. The questionnaire results are displayed on the slide in real time, which can help presenters to grasp opinions about their presentation. Because users can make their questionnaires in PowerPoint, doing so is quite easy. This paper explains the development of the system and demonstrates that the real-time questionnaire system has high performance and scalability.
\end{abstract}

Keywords-Interactive presentation; real-time Web questionnaire; collaborative tools; communication aids; information sharing; Web services

\section{INTRODUCTION}

In an interactive presentation, a lecturer gives a presentation for an audience, which provides feedback about the presentation slide by slide. We proposed a hybird-interaction presentation system [1], [2], [3] that consists of three sub-systems: a presenter system, an audience system and a back-end system. The presenter system utilizes an existing presentation software (PowerPoint) to display presentation slide content but extends that software to include interactive presentation functions. The audience system is a Web application for collecting comments from the audience; it shows the same slide image along with the presenter's display. In this paper, we describe the implementation of the real-time questionnaire function and evaluate its performance.

We explain the implementation details of Silhouette Arena, which realizes the hybrid interactive presentation, and we evaluate the execution time of the real-time questionnaire function. The execution time is the time that elapses between when the presenter system accumulates the votes from audience and when the result graph is shown. The peak of the execution time is from $350 \mathrm{~ms}$ to $400 \mathrm{~ms}$.

We developed a novel interactive presentation system as a Web application containing a simple and powerful feedback subsystem. No presentation software supports the functions of an interactive presentation. The existing presentation software assumes that presenters will not edit presentation slides or add slide show objects without using the editor window, even when giving a presentation. To easily develop this functionality, we implement a co-application of PowerPoint to utilize existing software functions (e.g., making shapes or displaying bar graphs). The real-time questionnaire function uses existing presentation software functions to display the questionnaire result as a line graph, pie chart or bar graph.

The hybrid interactive presentation system provides the real-time questionnaire function to count votes and shows the results in the presentation software's slide show mode; the synchronizing slide content function displays the same slide along with the presenter's screen. The questionnaire function has a crucial part in the interactive presentations because it attracts the audience and increases the depth of listener's understanding of the presentations. In a decision-making scene, the questionnaire function is utilized as a supporting tool when making a decision.

In our previous papers, we have not presented the whole architecture of the system; thus, this paper presents the architecture of a hybrid interactive-presentation system, called Silhouette Arena.

The paper is organized as follows. In Section II, we discuss the interactive presentation. In Section III, we explain a design goal of the real-time questionnaire function. In Section IV, we explain the interactive presentation system. In Section V, we describe the implementation of the real-time questionnaire. In Section VI, we show the evaluation of the real-time questionnaire function. In Section VII, we discuss the evaluation and the system's performance. In Section VIII, we conclude the paper and summarize the effects of real-time voting.

\section{INTERACTIVE PRESENTATION}

An interactive presentation support system is required in education because Questions and Answers are important in classrooms. For instance, clicker devices are employed in education for interactive presentations. This section describes hardware and software clickers and their limitations in education.

\section{A. Software/Hardware clicker}

Hardware clickers are widely used in classes. Clickers are handheld devices. By using clickers with wireless systems, professors can ask questions, and students can respond in a 


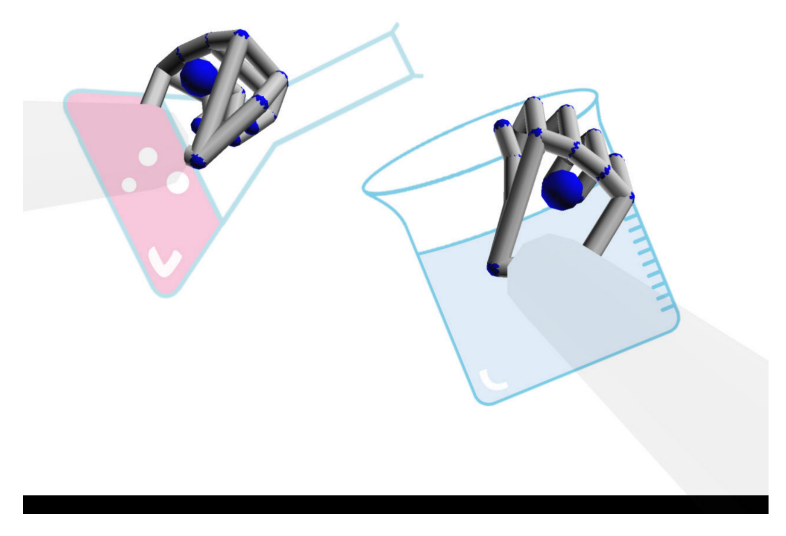

Fig. 1: Presenter can manipulate slide objects with motion controllers during an ongoing presentation.

timely manner [4], [5]. Clicker assessment and feedback (CAF) is an instructional assessment and feedback strategy that is incorporated into interactive technologies for higher education; these technologies are often referred to as clickers. CAF is important as one aspect of the interactive presentation. Existing studies of CAF confirm the efficacy of the its interactive functionality for presentations in education, but the handheld devices for gathering feedback from students are a hindrance in a large class.

Software clickers have been used to avoid the trouble of having to install specific physical devices. Hauswirth et al. proposed a software clicker to teach Java programming for higher education. Their software clickers provided much richer types of problems than just the simple, traditional multiple-choice questions [6]. Employment of software clickers eliminates the burden of installing devices; however, the software must be configured before each presentation to collect opinions.

\section{B. Web application}

In general, Web applications can resolve the configuration problem. Triglianos et al. proposed an interactive presentation system as a Web application [7]. However, the proposed method provides less flexibility for designing presentation materials than can be obtained by using the conventional presentation applications such as PowerPoint.

Generally, it is impossible to perfectly predict the knowledge of an audience or to answer questions ahead of time. Designing presentation slides and giving presentation are challenging when any aspect of the situation, such as the place, the audience's knowledge or any of the questions that will be asked, is unexpected. Lecturers need a system to help deliver presentations in an unexpected context. For flexible presentations, Kanev [8], [9] proposed tangible interfaces based on direct interactions with digitally encoded versions of printed handouts.

We are developing the Silhouette Effects system, which is dedicated to scaling conventional presentation tools to create instant manipulations of slide objects while in the presentation mode [10]. Fig. 1 shows that a presenter using Silhouette
Effects can manipulate slide objects with motion controllers during an ongoing presentation. Silhouette Effects supports only the features needed for delivering presentations. We have been developing a hybrid interactive presentation system that is intended to improve presentations by allowing interaction between lecturers and audiences without losing the flexible presentation functionality. This hybrid interactive presentation system extends Silhouette Effects. We demonstrate a real-time questionnaire system that is based on the hybrid interactive presentation system and that gathers feedback from audiences in presentations using HTML5 Web technologies.

\section{REAL-TIME QUESTIONNAIRE FOR INTERACTIVE PRESENTATIONS}

Our system has a real-time questionnaire function that enables a lecturer to collect what audiences are thinking about the presentation using print questionnaires. In this section, we define the general requirements and the functions needed to realize the real-time questionnaire.

\section{A. Requirements}

Our goal is to realize a simple method for creating a realtime questionnaire, which is the type of the questionnaire in which the result is displayed on a shared screen in real time during the implementation of a presentation. Having a real-time questionnaire in a presentation helps to maintain the audience's focus on the presentation and increases the depth of the audience's understanding of the presentation. In conventional research, specific devices are needed to gather answers from audiences. Our approach requires that the answers can be collected using a Web application, which simplifies the operation of performing the real-time questionnaire.

Our objective is for the system to provide seamless questionnaires as part of an interactive presentation without extra efforts. Moreover, the questionnaire system should be scalable to match the audience size. We suppose that a large number of audience members will use the real-time questionnaire at almost the same time. Hence, we must consider the scalability of the real-time questionnaire. Increasing requests to the system leads to network congestion and high processing times, which can cause delays when displaying the results of the questionnaires in real time. Moreover, because of the requirements of the specific devices, as expressed in the approaches of the previous studies, the effort needed to carry out a questionnaire is extensive when audience is large, as the system does not scale well.

\section{B. Design Goals}

One of the goals of the Silhouette Arena, a hybrid interactive presentation system, is to provide a real-time questionnaire in an interactive presentation when the lecturer and the audience are in the same location. Our objectives during the development of the Silhouette Arena are to ensure interoperability, interactive functionality, and scalability.

Interoperability means that users can answer questionnaires on their own devices. This helps administrators to reduce the cost of preparation. If the system requires special devices for presentations, setup can be time-consuming to set up them. To ensure interoperability, we develop a Web application for 


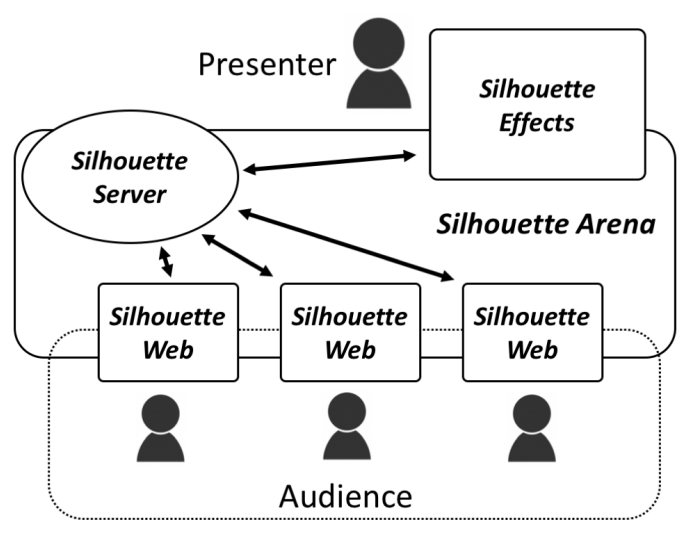

Fig. 2: Silhouette Arena: Hybrid Interactive Presentation System.

the audience. This approach has two merits. The first is that it is not necessary to create specific devices and applications for collecting comments and opinions from audience members. The second merit is that personal devices such as notebooks, smart phones and tablet computers can be used in our system.

Interactive functionality refers to real-time communication between the lecturer and the audience. For example, the lecturer and the audience can share presentation slides, and the lecturer can learn the results of a questionnaire in real time while delivering a presentation. The audience can provide feedback through questionnaires during presentations, which helps to improve the communication among participants. Our system supports real-time information presentation for lecturers, including feedback from audience members. The system supports interactive functionality to ensure communication between the lecturer and the audience during the real-time questionnaire. The system presents the results of the questionnaires on a shared screen using graphs from Microsoft Excel. To update the results of the presentation slide in accordance with the received answers, the audience members share presentation slides in real time using a Web application operating that runs on general Web browsers.

Scalability refers to the need to realize a system that can summarize a questionnaire and display the results in real time even if a large number of audience members participate in a presentation. The Web application for the audience contributes to extending the scalability of our system because it allows independence from using specific devices for gathering feedback. Specifically, we need to avoid network congestion and the high processing time when there are a large number of responses from the audience. We developed a tallying server to reduce the load of a system on the presenter's system. We investigated the optimal frequency for updating graphs on presentation materials according to frequent requests.

\section{Silhouette ARENA: SyStem FOR HYbrid INTERACTIVE PRESENTATION}

\section{A. Architecture}

The architecture of the hybrid interactive presentation system called Silhouette Arena is shown in Fig. 2. Silhouette

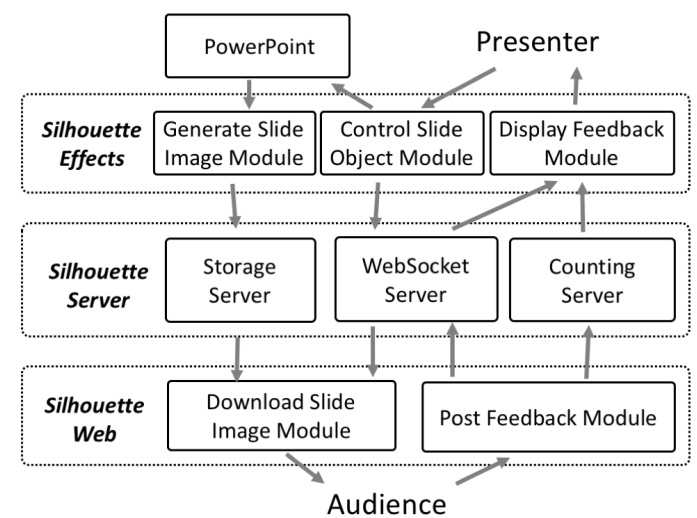

Fig. 3: Architecture of Silhouette Arena.

Arena consists of three main parts: Silhouette Effects, Silhouette Web and Silhouette Server (see Fig. 2).

Silhouette Effects controls a PowerPoint to share presentation slides with clients called Silhouette Web. Silhouette Effects provides the presentation with supporting features, such as the ability to send the information on shared presentation slides. Moreover, Silhouette Effects enables users to manipulate slide objects. The presentation controller manipulates the position and size of slide objects, such as text and drawings, during the presentation slide show mode of PowerPoint. The lecturer can collect electronic questionnaires from audience members during the presentation. When an audience member sends a questionnaire, the totals across the questionnaires are expressed using an Excel pie chart during the presentation. While audience members submit answers to the questionnaire, the results are visualized in real time using Excel graphs on a presentation slide.

Silhouette Web has a user interface for showing a slide or for adding annotations and free-form handwritten memos on the slide. The audience can add their opinions into the slide and share their opinions with the other participants, including the presenter. The Silhouette Web has two visual layers. The first layer, the background of the Web application, shows a presentation slide; the Web application's background image synchronizes to the presenter's slide show screen. This approach is known to perform reliably and accurately, as presentation slides have often been converted into images using Web conferences in previous research [11]. The second layer shows the user annotation objects that are shared by the audience members.

Fig. 3 shows the architecture of the system. Silhouette Effects communicates with Silhouette Web via Silhouette Server.

Silhouette Server consists of three internal subsystems. The first subsystem is a storage server for the presentation slides that have been converted into images. The presentation controller creates the slide images from the presentation slides and uploads the images to the storage server when the lecturer starts the presentation slide show. The storage server assigns a unique URL to each uploaded image and stores that image using the file system storage. 


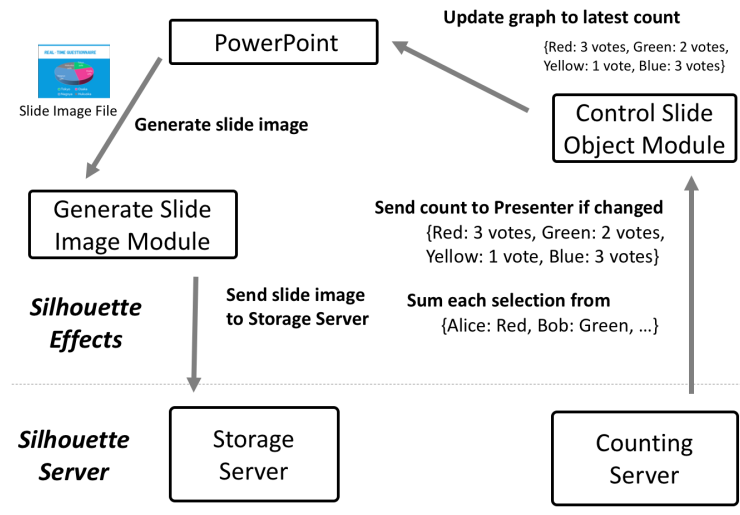

Fig. 4: Control Flow of Updating Pie Chart.

The second subsystem is a WebSocket server for real-time communication among the participants of the presentation, lecturer and the audience members. The subsystem relays received data in real time to other participants. Silhouette Effects notifies a list of the uploaded image URL via the subsystem. When an audience member creates a new annotation or handwritten memo, Silhouette Web sends the new data to Silhouette Effects via Silhouette Server. Silhouette Effects creates a new slide object from the received data and appends that object to the shown slide in real time. Then, the feedback from audience members is displayed on the presentation slides in real time.

The third subsystem is the counting server for the realtime questionnaire. This subsystem aggregates the electronic questionnaire responses and creates a pie chart with the data. The subsystem manages the real-time questionnaire using the following three steps. First, Silhouette Effects notifies all Silhouette Web users about the start of the real-time questionnaire, and then Silhouette Web shows the answers of the vote. Second, Silhouette Web sends the answer that each audience member selected to the subsystem; the subsystem then tallies the answers and saves the result to storage. Third, the subsystem periodically reports the latest result to the presentation controller.

Fig. 4 presents how to update a pie chart with PowerPoint material using Silhouette Arena to prevent unexpected crashes of the PowerPoint system. The most important part is the counting server in Fig. 4. The counting server keeps pace without crashes of the PowerPoint by updating the pie chart. Then, after the counting server receives voting data from Silhouette Web clients, it collects the votes and periodically sends the result to the Control Slide Object Module. When the Control Slide Object Module receives the voting result, it uses PowerPoint to update the graph and sends an image file of the slide to Silhouette Server to distribute the image to the clients.

\section{B. Silhouette Effects: System for Enhancing Presentation Ex- pressions}

Our approach helps the lecturers to give a presentation effectively using Silhouette Effects. There are two modes in Microsoft PowerPoint: a designing mode for modifying slide objects and a presentation mode for giving a presentation; normally, the users cannot edit slide objects when in the presentation mode. Silhouette Effects expands the methods that can be used in the presentation mode. When delivering a presentation, running our system along with PowerPoint allows a user to modify slide objects at while in presentation mode.

The proposed method for manipulating objects on slides in real time leads to a reactive presentation. Manipulating slide objects by moving, zooming and adding visual effects allows lectures to guide the audience and keep the its attention, as well as to communicate information in a visual space by presenting animations and slide object effects reactively. Moreover, Silhouette Effects provides presentation-supporting functions other than the manipulations of slide objects; these include handwritten memo and pointing features, and they are available in the presentation mode.

The lecturers perform the presentation-supporting functions with input operations from a mouse and a keyboard or with gesture inputs from Leap Motion or Kinect. Because Leap Motion and Kinect have an advantage over the typical input devices, such as a mouse and a keyboard, our system corresponds best with the gesture input interface. Leap Motion supports very precise finger tracking, so we constructed a highly intuitive interface using Leap Motion. Additionally, Kinect has high user mobility and supports skeleton tracking in a wide recognizable area. Therefore, using Kinect, we constructed an interface with high user mobility that can interact through the movement of users' whole bodies. The gesture input interface for manipulating slide objects supports gestures bound to mundane actions such as grasping something, and these gestures are widely known; the include swiping and pinching in or out. Thus, the gesture input interface using Leap Motion and Kinect achieves seamless input operations for the presentation-supporting functions.

\section{Silhouette Web: Feedback System for the Audience}

Fig. 5 shows Silhouette Web, the user interface of the Web application for submitting feedback from audience members.

The audience members open the Web application, Silhouette Web, on the Web browser to participate in the presentation. Silhouette Web is available on modern Web browsers that support HTML5. Next, we describe the user interface of the Silhouette Web in detail.

To use the real-time questionnaire, the audience members need to enter an ID code. The counting server manages the votes according to users' ID codes and tallies them. If an audience member sends a vote twice, the counting server will only tally the last vote, so audience members can change their votes as long as the real-time questionnaire is in session.

The four-colored buttons in the bottom-left of the screen are used to vote in the real-time questionnaire session. The left and right arrow buttons are used to change to the previous or next slide. The slider between the arrow buttons is also used 


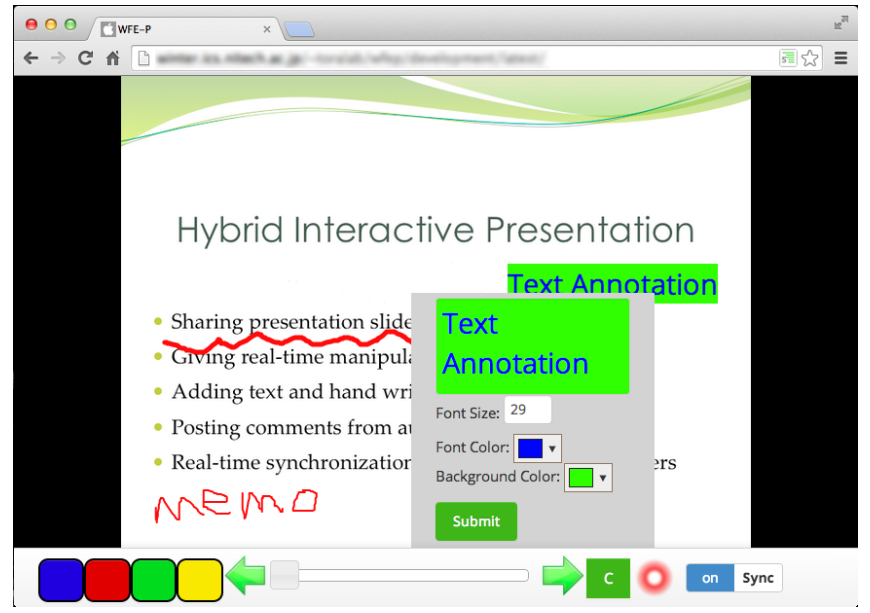

Fig. 5: In the Silhouette Web application, the audience can provide feedback, text annotations and freehand drawings.

to change the slide. The "C" button is for posting comments. The switch button on the bottom-right of the screen is used to change the synchronization mode. In the questionnaire session, when the audience can click one of the four-color buttons, Silhouette Web sends the answers to Silhouette Server. If the comment-posting button is clicked, a comment form window is shown. Then, the audience member inputs a comment in the window and sends the comment to the Silhouette Effects, which shows the newly received comment on the slide and automatically saves the comment into the presentation slide file. The switch button is for turning the synchronization mode on or off depending on whether Silhouette Web synchronizes with the lecturer slide in real time or not. After a presentation, the lecturers check the submitted comments to assess the audience's feedback. The synchronization on/off button configures whether other users' manipulations are synchronized in real time. When the synchronization is off, the lecturer's and audience's are not synchronized. This button allows the users to genuinely browse the presentation slides.

The audience members can move the pointer by pressing and holding the mouse button and dragging the mouse. Rightclicking on presentation slides displays the dialogue for adding an annotation (see Fig. 5). The annotations are managed using DOM elements that handle the user's operation, so the audience member can edit and move the existing annotations on the slide.

\section{IMPLEMENTATION OF REAL-TIME QUESTIONNAIRES}

\section{A. Mechanism of the Real-Time Questionnaire}

In the real-time questionnaire session, Silhouette Web and Silhouette Effects communicate via the counting server, Silhouette Server.

Silhouette Web sends the answers to the questionnaire as JSON data. The JSON data contain the ID code to identify the person who voted and the code of the selected button. The counting server updates the results when a new answer is received.

The counting server periodically notifies the presentation

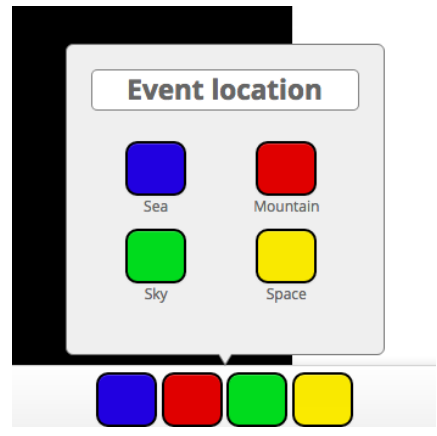

Fig. 6: Vote interface.

controller of new results at an interval of two seconds. The direct way that Silhouette Web sends answers to Silhouette Effects create a heavy processing load on the network traffic. This processing load is one of hindrance of the presentation. To suppress the processing load, our approach uses an external server to count the results of the questionnaires, and the external server sends the results to the Silhouette Effects periodically.

Our system enables users to share questionnaire results in real time. Sharing questionnaire results requests two steps. First, when receiving a questionnaire result, the presentation controller updates a pie chart that displays the results using Excel. Then, the presentation controller uploads the image of the presentation slide that contains the updated graph and broadcasts the URL of the slide image as JSON data to Silhouette Web through Silhouette Server. After Silhouette Web updates the image on the first layer (which displays presentation slides with the received image), the result of the questionnaire is shared in real-time.

\section{B. Real-Time Web Questionnaire Protocol}

The interactive presentation provides the real-time questionnaire system that enables a lecturer to publish an electronic questionnaire to audience members and to collect the answers during the presentation. In this section, we describe a messaging protocol for the real-time questionnaire system.

Questionnaire session management in the real-time questionnaire system consists of six steps: announcement, opening, assignment, waiting, closing and cleanup. In the first step, the system broadcasts to the clients a title and the options as part of a vote session, and the clients display the title and the options. The title is labeled 'Event location' in Fig. 6. The buttons on the client display the options; these are 'Sea', 'Mountain, 'Sky' and 'Space' in Fig. 6. The system does not accept any votes at this time. In the second step, the system begins to accept votes from the audience devices. In the third step, the system assigns unique numbers to each client to identify the voters. In the fourth step, the system waits for votes. The clients send the selections with assigned user numbers. In the fifth step, the system closes the vote session. The system finishes accepting votes, and the clients disable the buttons on the audience devices. In the last step, the system releases the unique numbers to prevent the tracking of voters. Finally, the system reveals the results. 
Next, we explain the protocol messages for each session. In the first step, the system uses a Prepare Vote Notification Message. In the second step, the system uses an Open Vote Notification Message. In the third step, the system uses a Vote Request Message and a Vote Response Message. In the fourth step, the system uses a Close Vote Notification Message. In the last step, the system uses a Result Notification Message. Below, we explain these messages in detail.

- Prepare Vote Notification Message: This message has three fields: The first is a vote session ID, the second is a vote session title, and the third consists of pairs of selection item IDs and labels. The audience system updates the selection button labels of the UI upon receiving the message.

- Open Vote Notification Message: This message has three fields: The first is a vote session ID, the second is a time stamp of when the message was sent from the presenter's system, and the third is a temporary unique number. The client should include the number in a Vote Request Message as described below. The message should occur once per vote session. The audience system enables the selection buttons of the UI when the message is received.

- Close Vote Notification Message: This message has two fields: The first is a vote session ID, and the second is a time stamp of when the message was sent from the presenter's system. The message should occur once per vote session. The audience system disables the selection buttons of the UI when the message is received.

- Vote Request Message: This message is sent from the client system and has four fields: The first is a vote session ID, the second is a time stamp of when the message was sent from the audience member's system, the third is a temporary unique number, and the fourth is a selection ID. The message should occur one or more times in each vote session. The message is available if the Open Vote Notification Message has been received and if the Close Vote Notification Message has not been received for the same vote session ID.

- Vote Response Message: This message is sent from the server system in response to the Vote Request Message. This message has four fields: The first is a vote session ID, the second is a time stamp of when the message was sent from the server system, the third is a temporary unique number, and the fourth is a response code that indicates whether the request has been accepted.

- Result Notification Message: This message is sent periodically from the presenter's system to display the current selection count on the audience devices. This message has three fields: The first is a vote session ID, the second is a time stamp of when the message is sent from the presenter's system, and the third is an array of selection counts in the same containing the pairs of selection item IDs and labels from the Prepare Vote Notification Message. This message broadcasts the

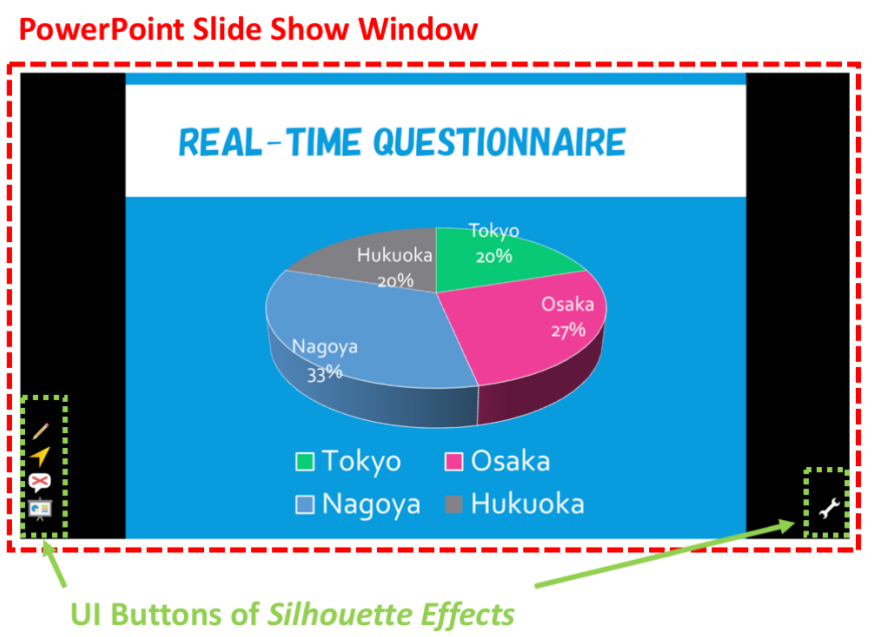

Fig. 7: The Silhouette Effects system uses PowerPoint to display questionnaire results.

current selection status in the questionnaire session. The vote session ID is valid from when the vote session begins until the session is closed.

\section{Displaying Results of Real-Time Questionnaires}

Generally, changing the shape object attributes (e.g., coordinating position in a slide or changing background color of the shape) on a slide at a high frequency requires high CPU loads. To suppress these high loads, the graph update module has a minimum interval for updating the graph's properties. In the experimental case, we fixed the minimum interval at 1 second.

Fig. 7 is a screenshot displaying the questionnaire results on the device of a lecturer. The presentation controller icons are located in the lower-left and lower-right corners, as shown in Fig. 7; this is the interface of Silhouette Effects which helps lecturers in their presentations. Silhouette Effects expands the methods for using Microsoft PowerPoint to modify slide objects such as text, graphs and figures, while in the presentation slide show mode. The presentation controller provides the presentation-supporting features and sends the information needed to share the presentation slides to Silhouette Server.

Lecturers can start a questionnaire at any time during presentations by using the presentation controller. Because our real-time questionnaire supports up to four alternatives, the lecturer can configure the alternatives using the presentation controller. Then, a graph such as the one in Fig. 7 is generated to display the result of the questionnaire in real-time. The presentation controller inputs the lecturer's configuration of the alternatives into a Microsoft Excel spreadsheet and visualizes the configuration as a graph object. The graph object in Excel gets copied into the PowerPoint presentation. When receiving the results of questionnaires from the counting server that tallies the audience answers, the presentation controller passes the results to the Excel spreadsheet (which is running in the presentation background), and the graph object visualizes the result. When receiving the second and subsequent results, 
the presentation controller rewrites the data in the Excel spreadsheet, and the graph object is then updated in real time.

Specifically, the presentation controller saves the questionnaire results as PowerPoint graphs in the presentation file. Therefore, the lecturer can refer to the results after the presentation without effort. For a general conference, the results need to be recorded in the conference notes using for future reference. The real-time questionnaire system avoids the trouble of recording by automatic saving.

We propose a hybrid interactive presentation system that connects the traditional presentation-supporting tool and the Web application to gather feedback from audience members. The hybrid interactive presentation system occupies an important role as the basis of the real-time questionnaire system.

\section{Preparation before Presentation}

To use the real-time questionnaire function in the presentation slide show, the presenter prepares a slide and a graph object using the following steps: First, add a new slide if needed. Second, add a graph object (such as a pie chart or bar graph) on the slide. Finally, set the graph label title using the Excel cell editor.

In the presentation slide show, the presenter's system checks whether a slide has a graph object when the slide is shown for the first time. If the slide has such an object, the presenter's system sends a Prepare Vote Notification message and an Open Vote Notification message to the audience system via the server system. The presenter's system automatically sets the graph label colors that correspond to the client Web application's vote buttons.

\section{EVALUATION}

In this section, we describe the procedure for evaluating the real-time questionnaires function and the result of that evaluation. We measured the execution time needed to update the graph parameters.

\section{A. Experimental Procedure and Environment}

We made an evaluation script to measure the execution time for updating the graph parameters. First, the script opens a PowerPoint presentation file that has a slide containing a pie chart for the real-time questionnaire. Next, the script performs the following steps 100 times after pre-execution and 10 times for warm-up. For the first step, the script records the start time $\left(t_{0}\right)$. For the second step, the script generates four random numbers and updates four graph parameters on the slide graph. For the third step, the script records the end time $\left(t_{1}\right)$. Finally, the script closes the file. The execution time is calculated by $t_{1}-t_{0}$. We used a MacBook Pro for the presenter's device; it has an Intel Core i7 $2.3 \mathrm{GHz}$ processor, $16 \mathrm{~GB}$ of $1,600 \mathrm{MHz}$ DDR3 RAM, Windows 10, and PowerPoint version 16.0. We performed the script 10 times; therefore, we sampled 1,000 execution times.

\section{B. Experimental Result}

Fig. 8 shows the execution-time histogram for the updating graph parameters. We sampled 1,000 execution times. In Fig.

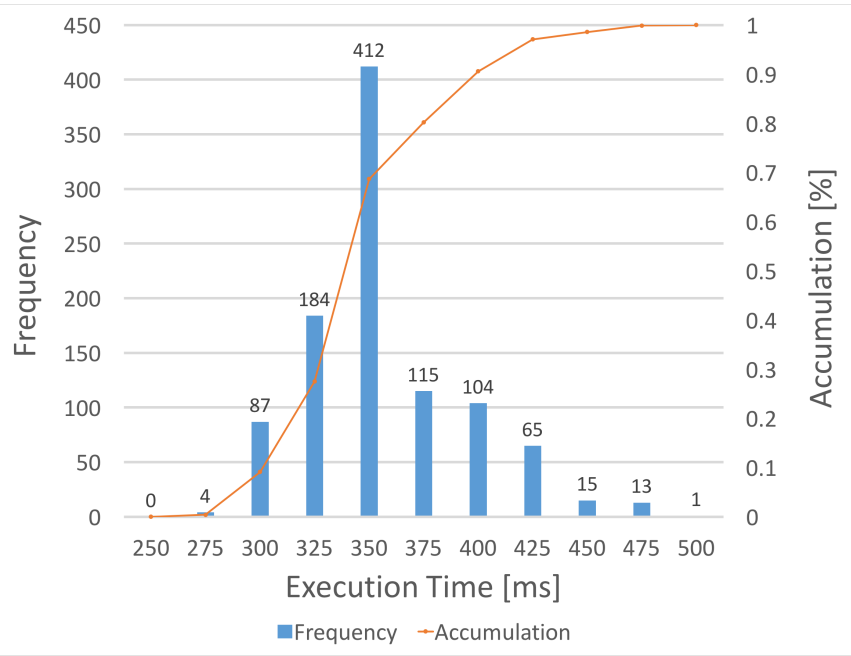

Fig. 8: Execution time histogram for an updating pie chart.

8, the horizontal axis indicates the sampling period for the execution time, the left vertical axis indicates the frequency, and the right vertical axis indicates the accumulation of the frequency. The peak appears between the $350 \mathrm{~ms}$ and $375 \mathrm{~ms}$ sampling periods, $90 \%$ of the execution times are below 425 $\mathrm{ms}$, and all the execution times are below $525 \mathrm{~ms}$. Therefore, the slide object controller can update the graph parameters about twice per second.

\section{DISCUSSION}

In this section, we discuss the usability of Silhouette Arena, the system from point of view for decision support and the security of the real-time questionnaire.

\section{A. Usability}

Silhouette Arena enables the lecturer and audience members to communicate about the presentation contents during a presentation. Silhouette Arena's subsystem for presenter, Silhouette Effects, extends the existing software (PowerPoint). The subsystem for the audience, Silhouette Web, is a simple Web application. These system reduce the time and expense needed when learning to use Silhouette Arena because the lecturer uses existing presentation software to change slides and modify slide objects. The audience members submit their comments through the simple user interface that is illustrated in Fig. 5.

The accumulated result of the votes is visualized as a graph object (e.g., a bar graph or a pie chart) on a presentation slide using Excel functions. To extend the presentation controller, the lecturers can perform complex questionnaires which let the audience members to choose multiple options from the choices.

\section{B. Decision Support}

We discuss the applicability of our system for decision support. First, we explain the system's scalability. Second, we consider its usability in decision-making. 
Our interactive presentation system can be applicable to larger-scale presentations (e.g., ordinary conferences in enterprises and organizations). The experimental result proves that the real-time questionnaire system is efficient and scalable for use as a feedback mechanism at large conferences. We conclude that users can operate the real-time questionnaire system in ordinary conferences and in enterprises and organizations.

Our system is helpful not only in education but also in decision-making. Let us discuss the system from point of view of the application as the decision-making system. The decision-making system supports various methods of decisionmaking, such as brainstorming and the KJ Technique, so the participants offer their opinions in the same place and organize their opinions using any type of procedure. The posting feedback feature allows for the collection of users' opinions and other data as a key step; the submitted opinions and data are arranged on the shared presentation screen. Furthermore, when using the real-time questionnaire system, the users effectively perform decision-making techniques and forge a convergence of opinion.

First, specifically, our annotation function enables users to store their opinions on the presentation slides. Drag-anddrop annotations and handwritten memos are synchronized in real time, and the features are supportive of various methods of decision-making, such as grouping opinions using the $\mathrm{KJ}$ Technique. The operations are performed on the shared screen, and users deal with the operations cooperatively.

Second, the real-time questionnaire is available to summarize options and reach an agreement when gathering and organizing opinions. This real-time visualization of the questionnaire results has the potential to impact the process of decisionmaking; for instance, the users can be influenced by other people's opinions. The real-time questionnaire can prompt the users to defer to or to deprecate other users' answers votes. An approach that integrates the real-time questionnaire into traditional decision-making methods could be used to develop a new methodology of decision-making.

Compared with the traditional system for supporting decision-making, our system widens the scope of participants, as the interactive presentation functions successfully for a large group. Because the functions for submitting opinions provide anonymity, the users can submit their opinions at ease, which stimulates discussions. The real-time questionnaire that existing applications lack improves the lecturer's ability to form an opinion about the discussions.

\section{Security}

Naturally, security is an important issue for these questionnaires. For example, cheating by selecting multiple responses must be prevented and assurance of anonymity is required. Therefore, the real-time questionnaire system should be independent of certain troublesome coded communication channels and authentication mechanisms so as to ensure safety and anonymity in questionnaires.

However, security was not our concern in this study. We tried to realize the efficacy of decision-making at a certain scale of meetings in enterprises and organizations. As users fill out the questionnaires, they want to feel safe; thus, we implement a new questionnaire mode for tackling the problems with existing approaches [12].

\section{CONCLUSION}

We proposed the interactive presentation system, Silhouette Arena, which provides efficient, real-time questionnaire functions to collect feedback from presentation attendees. The system helps presenters to create a sophisticated presentation through the slide-object-manipulation function in Silhouette Effects. Moreover, Silhouette Web helps audience members to provide real-time feedback on presentations. We explained how the implementation of the system improves the efficiency of the real-time questionnaire function. We demonstrated the efficiency of the system, finding that the system can be used at a large conference, in an organization or in a class. The approach of using a hybrid interactive presentation system contributes to the establishment of a new type of presentation.

\section{ACKNOWLEDGMENT}

This work was supported in part by JSPS KAKENHI Grant Number JP15K00422, JP16K00420.

\section{REFERENCES}

[1] Ryota Inoue, Shun Shiramatsu, Tadachika Ozono, Toramatsu Shintani, Visualizing Real-Time Questionnaire Results to Promote Participation in Interactive Presentations, Proc. of the 2014 IIAI 3rd International Conference on Advanced Applied Informatics (IIAI-AAI 2014), pp.6469, 2014.

[2] Ryota Inoue, Shun Shiramatsu, Tadachika Ozono, Toramatsu Shintani, An Interactive Presentation System Based on Feedback to a Presentation Material of an Ongoing Presentation, IPSJ Journal, Vol.56, No.10, pp.2011-2021, 2015.

[3] Yusuke Niwa, Shun Shiramatsu, Tadachika Ozono, Toramatsu Shintani, An Efficient Method for Distributing Animated Slides of Web Presentations, International Journal of Advanced Computer Science and Applications(IJACSA), Vol.7, Issue 1, pp.612-620, 2016.

[4] S. M. Keough, Clickers in the Classroom A Review and a Replication, Journal of Management Education, Vol.36, No.6, pp.822-847, 2012.

[5] J. H. Hana and A. Finkelsteinb, Understanding the effects of professors' pedagogical development with Clicker Assessment and Feedback technologies and the impact on students' engagement and learning in higher education, Journal of Computers \& Education, Vol.65, pp.64-76, 2013.

[6] M. Hauswirth and A. Adamoli, Teaching Java programming with the Informa clicker system, Journal Science of Computer Programming, Vol.78, pp.499-520, 2013.

[7] V. Triglianos and C. Pautasso, ASQ: Interactive Web Presentations for Hybrid MOOCs, Proceedings of the 22nd international conference on World Wide Web companion, pp.209-210, 2013.

[8] K. Kanev, Tangible interfaces for interactive multimedia presentations, Journal of Mobile Information Systems, Vol.4, No.3, pp.183-193, 2008.

[9] B. Signer and M. C. Norrie, PaperPoint: a paper-based presentation and interactive paper prototyping tool, Proceedings of the 1st international conference on Tangible and embedded interaction, pp.57-64, 2007.

[10] Hiroyuki Yamada, Shun Shiramatsu, Tadachika Ozono, Toramatsu Shintani, A Reactive Presentation Support System based on a Slide Object Manipulation Method, Proceedings of The 2014 International Conference on Computational Science and Computational Intelligence, Vol.2, pp.4651, 2014.

[11] H. Louafi, S. Coulombe and U. Chandra, Efficient Near-Optimal Dynamic Content Adaptation Applied to JPEG Slides Presentations in Mobile Web Conferencing, Proceedings of the 27th international conference on Advanced Information Networking and Applications, pp.724-731, 2013.

[12] Y. Goto, Information Assurance, Privacy, and Security in Ubiquitous Questionnaire, Proceedings of the fourth international conference on Frontier of Computer Science and Technology, pp.619-624, 2009. 